\title{
Some thoughts concerning power sums
}

\author{
Dedicated to the memory of Zoltán Szvetits (1929-2014) \\ Gábor Nyul* \\ Institute of Mathematics, University of Debrecen \\ H-4010 Debrecen P.O.Box 12, Hungary \\ e-mail: gnyul@science.unideb.hu
}

April 27, 2015

\begin{abstract}
In this note we present an elementary way to derive directly closed-form expressions for power sums. Applying this method, we deduce some general results on power sums with arbitrary exponents. Finally, we give an outlook on higher mathematical connections between power sums, Stirling and Bernoulli numbers.
\end{abstract}

2010 Mathematics Subject Classification: 11B68, 11B73

Keywords: power sums, Stirling numbers, Bernoulli numbers

\section{Introduction}

Zoltán Szvetits was a legendary teacher, one of the founders of special mathematics education in Mihály Fazekas High School, Debrecen, 50 years ago. I have had the distinct blessing to know him personally. When I started my studies in the first six-year class in this school, the same school year was the very last for Mr. Szvetits before finally retiring.

Once he heard that I learnt mathematical induction. He asked my teacher to let me miss the mathematics lesson. We sat into the library, and he taught me that although induction is a powerful tool, it has some disadvantages. First, one has to know or at least conjecture the statement to be proved. On the other hand, in many cases, proof by induction needs no or essentially no beautiful ideas.

If $k$ and $n$ are positive integers, we shall use the notation

$$
S_{k}(n)=1^{k}+\ldots+n^{k}
$$

for power sums.

\footnotetext{
${ }^{*}$ Research was supported in part by Grant 100339 from the Hungarian Scientific Research Fund.
} 
Typical basic exercises are proving by mathematical induction that the sums of the first few positive integers, square numbers and cube numbers are

$$
\begin{gathered}
S_{1}(n)=1+\ldots+n=\frac{n(n+1)}{2}, \\
S_{2}(n)=1^{2}+\ldots+n^{2}=\frac{n(n+1)(2 n+1)}{6}, \\
S_{3}(n)=1^{3}+\ldots+n^{3}=\frac{n^{2}(n+1)^{2}}{4} .
\end{gathered}
$$

Indeed, it is almost automatic to prove these identities by induction.

In this note we show that ingenious idea which I have learnt from Mr. Szvetits and which gives a direct, elementary approach to identities (1), (2), (3). Up to this day, I treasure his handwritten papers. It is typical of him that he used the notation $S_{n}^{\ominus}$ for $S_{3}(n)$.

Thereafter, we think over this method to obtain some general results on $S_{k}(n)$. Finally, we close our paper with an outlook on the connections of these expressions with Stirling and Bernoulli numbers.

\section{Sums of squares and cubes}

Everybody knows the anecdote about the schoolchild Gauss who calculated $S_{1}(100)$ rapidly, surprising his teacher. To explain his idea, write $S_{1}(n)$ in reverse order

$$
S_{1}(n)=n+\ldots+1,
$$

and add it to $S_{1}(n)$ in the original order of terms to get $2 S_{1}(n)=n(n+1)$, therefore (1) is proved.

In the next paragraphs we will consider identities (2) and (3), but we should mention that the same argument can be also applied to obtain (1).

The first $n+1$ positive cube numbers can be written in the following way:

$$
\begin{array}{rrrrr}
(0+1)^{3}= & & 1 \\
(1+1)^{3}= & 1^{3}+3 \cdot 1^{2}+ & 3 \cdot 1+ & 1 \\
(2+1)^{3}= & 2^{3}+3 \cdot 2^{2}+ & 3 \cdot 2+ & 1 \\
& & & & \\
(n+1)^{3}= & n^{3}+3 \cdot n^{2}+ & 3 \cdot n+ & 1
\end{array}
$$

Summing up these equations, we get

$$
S_{3}(n)+(n+1)^{3}=S_{3}(n)+3 S_{2}(n)+3 S_{1}(n)+(n+1) .
$$

After some rearrangement and using (1), we have

$$
S_{2}(n)=\frac{1}{3}\left((n+1)^{3}-(n+1)-3 S_{1}(n)\right)=\frac{n(n+1)(2 n+1)}{6} .
$$


Similarly, write the fourth powers of the first $n+1$ positive integers as

$$
\begin{aligned}
& (0+1)^{4}=1 \\
& (1+1)^{4}=1^{4}+4 \cdot 1^{3}+6 \cdot 1^{2}+4 \cdot 1+1 \\
& (2+1)^{4}=2^{4}+4 \cdot 2^{3}+6 \cdot 2^{2}+4 \cdot 2+1 \\
& (n+1)^{4}=n^{4}+4 \cdot n^{3}+6 \cdot n^{2}+4 \cdot n+1
\end{aligned}
$$

Summation of these equations gives

$$
S_{4}(n)+(n+1)^{4}=S_{4}(n)+4 S_{3}(n)+6 S_{2}(n)+4 S_{1}(n)+(n+1) .
$$

From the previously proved identities (1) and (2), by an easy calculation it follows that

$$
S_{3}(n)=\frac{1}{4}\left((n+1)^{4}-(n+1)-4 S_{1}(n)-6 S_{2}(n)\right)=\frac{n^{2}(n+1)^{2}}{4} .
$$

\section{Power sums in general}

It is an immediate idea that the above argument applied in the general case allows us to derive an identity for $S_{k}(n)$ if we know the formulas for $S_{1}(n), \ldots, S_{k-1}(n)$. This will be carried out in the following theorem.

In addition, if we perform multiplications in (1), (2), (3), formulas

$$
\begin{gathered}
S_{1}(n)=\frac{1}{2} n^{2}+\frac{1}{2} n, \\
S_{2}(n)=\frac{1}{3} n^{3}+\frac{1}{2} n^{2}+\frac{1}{6} n, \\
S_{3}(n)=\frac{1}{4} n^{4}+\frac{1}{2} n^{3}+\frac{1}{4} n^{2}
\end{gathered}
$$

suggest us several observations on these expressions. It will turn out that our method is suitable to prove these properties, as well.

Theorem. $S_{k}(n)$ is a polynomial in $n$ with rational coefficients. The polynomial $S_{k}(x)$ possesses the following properties:

- the degree of the polynomial is $k+1$,

- the leading coefficient (the coefficient of $x^{k+1}$ ) is $\frac{1}{k+1}$,

- the coefficient of $x^{k}$ is $\frac{1}{2}$,

- the constant term is 0, i.e., 0 is a root of the polynomial, and

- -1 is also a root of the polynomial. 
Proof. Similarly as above, for the $(k+1)$ st powers of the first $n+1$ positive integers, binomial theorem gives

$$
\begin{aligned}
& (0+1)^{k+1}= \\
& (1+1)^{k+1}=1^{k+1}+\left(\begin{array}{c}
k+1 \\
k
\end{array}\right) \cdot 1^{k}+\left(\begin{array}{c}
k+1 \\
k-1
\end{array}\right) \cdot 1^{k-1}+\ldots+\quad\left(\begin{array}{c}
k+1 \\
1
\end{array}\right) \cdot 1+1 \\
& (2+1)^{k+1}=2^{k+1}+\left(\begin{array}{c}
k+1 \\
k
\end{array}\right) \cdot 2^{k}+\left(\begin{array}{c}
k+1 \\
k-1
\end{array}\right) \cdot 2^{k-1}+\ldots+\quad\left(\begin{array}{c}
k+1 \\
1
\end{array}\right) \cdot 2+1 \\
& (n+1)^{k+1}=n^{k+1}+\left(\begin{array}{c}
k+1 \\
k
\end{array}\right) \cdot n^{k}+\left(\begin{array}{c}
k+1 \\
k-1
\end{array}\right) \cdot n^{k-1}+\ldots+\quad\left(\begin{array}{c}
k+1 \\
1
\end{array}\right) \cdot n+1
\end{aligned}
$$

The sum of these equations is

$$
\begin{aligned}
& S_{k+1}(n)+(n+1)^{k+1}= \\
& S_{k+1}(n)+\left(\begin{array}{c}
k+1 \\
k
\end{array}\right) S_{k}(n)+\left(\begin{array}{c}
k+1 \\
k-1
\end{array}\right) S_{k-1}(n)+\ldots+\left(\begin{array}{c}
k+1 \\
1
\end{array}\right) S_{1}(n)+(n+1),
\end{aligned}
$$

which yields

$S_{k}(n)=\frac{1}{k+1}\left((n+1)^{k+1}-(n+1)-\left(\begin{array}{c}k+1 \\ 1\end{array}\right) S_{1}(n)-\ldots-\left(\begin{array}{c}k+1 \\ k-1\end{array}\right) S_{k-1}(n)\right)$.

Now, each of the properties listed in the theorem follows from this identity by induction on $k$.

Remark. By further consideration, it could be shown that

- $-\frac{1}{2}$ is a root of $S_{k}(x)$ if $k$ is even,

- 0 and -1 are multiple roots if $k \geq 3$ is odd.

\section{Stirling and Bernoulli numbers}

However, this is not the whole story. The polynomials $S_{k}(x)$ have a far-reaching theory. We highlight only two possible directions.

On the one hand, they can be expressed using Stirling partition numbers and Stirling cycle numbers as

$$
S_{k}(x)=\sum_{i=1}^{k+1} \sum_{j=i-1}^{k}(-1)^{j+1-i} \frac{1}{j+1}\left\{\begin{array}{c}
k \\
j
\end{array}\right\}\left[\begin{array}{c}
j+1 \\
i
\end{array}\right] x^{i}
$$

where $\left\{\begin{array}{c}m \\ \ell\end{array}\right\}$ counts the number of partitions of an $m$-element set into $\ell$ non-empty subsets, and $\left[\begin{array}{c}m \\ \ell\end{array}\right]$ denotes the number of permutations of $m$ elements which are the product of $\ell$ disjoint cycles $(\ell \leq m)$.

On the other hand, calculating the polynomials $S_{k}(x)$ for further small values of $k$, we can observe that the coefficient of $x^{i}$ is 0 if $i<k$ and they have the same parity. By a much deeper analysis, in the remaining cases it is more difficult to 
realize that the coefficient of $x^{i}$ is a constant multiple of $\frac{1}{k+1}\left(\begin{array}{c}k+1 \\ i\end{array}\right)$, which constant depends only on the difference of $k+1$ and $i$. Indeed, these polynomials satisfy Bernoulli's identity (sometimes called Faulhaber's identity)

$$
S_{k}(x)=\frac{1}{k+1} \sum_{i=1}^{k+1}\left(\begin{array}{c}
k+1 \\
i
\end{array}\right) B_{k+1-i} x^{i},
$$

where $B_{m}$ denotes the $m$ th Bernoulli number with the convention $B_{1}=\frac{1}{2}$. It is known that $B_{m}=0$ if $m \geq 3$ is odd. (Some authors define Bernoulli numbers such that $B_{1}=-\frac{1}{2}$. In this case Bernoulli numbers agree for any other indices, therefore the formula should contain an additional factor $(-1)^{k+1-i}$ inside the summation.)

For more details, one should consult the references listed below.

\section{References}

[1] T. Arakawa, T. Ibukiyama and M. Kaneko, Bernoulli Numbers and Zeta Functions, Springer, 2014.

[2] R. L. Graham, D. E. Knuth and O. Patashnik, Concrete Mathematics, Addison-Wesley, 1990.

[3] H. Rademacher, Topics in Analytic Number Theory, Springer-Verlag, 1973. 